\title{
BEAM LIFETIME STUDIES FOR THE SLS STORAGE RING
}

\author{
M. Böge, A. Streun, Paul Scherrer Institut, 5232 Villigen PSI, Switzerland
}

\section{Abstract}

SLS is a high brightness light source with $4.8 \mathrm{~nm}$ emittance at $2.4 \mathrm{GeV}$. Particle losses due to Touschek scattering will strongly affect the beam lifetime.

We present Touschek lifetime calculations based on a refined tracking procedure for determination of the lattice momentum acceptance. The dependency of lifetime on the gap height of insertion devices and on the RF cavity voltage is studied in detail for the reference optics of the SLS storage ring lattice.

Including gas scattering losses we expect $3.5 \mathrm{hrs}$ of total lifetime for $1 \mathrm{nCb}$ bunch charge and $0.1 \%$ coupling. Installation of a $3^{\text {rd }}$ harmonic cavity for bunch lengthening would increase the total lifetime to $8 \mathrm{hrs}$ and thus is recommended.

\section{TOUSCHEK TRACKING}

Touschek lifetime is approximately ${ }^{1}$ given as function of momentum acceptance (MA) and bunch volume integrated over the lattice structure $[6,12]$.

While the RF MA is given by the cavity voltage and almost constant along the lattice, the lattice MA depends on where the scattering event occurred and varies along the lattice. In particular we have to distinguish between

- non-dispersive sections, where a scattered particle will just follow the dispersive orbit, and local MA is determined by the momentum range of closed orbit existence or physical aperture, and

- dispersive sections, where a scattered particle will start an oscillation around the dispersive orbit, and local MA may be determined by dynamic aperture or by mismatch of dynamic to physical aperture.

Usual calculations [13] assume a perfectly linear and chromaticity corrected lattice and obtain the local MA from

$$
\delta_{a c c}^{L}\left(s_{o}\right)=\min _{i=1 \ldots N}\left\{\frac{a_{x i}}{\sqrt{H_{o} \beta_{x i}}+\eta_{i}}\right\}
$$

with $\delta:=\Delta p / p_{o}, H_{o}$ the lattice invariant (dispersion's emittance) at scattering location and $\beta_{x i}, \eta_{i}, a_{x i}$ horizontal beta function, dispersion and vacuum chamber half width at other lattice locations.

In modern light sources, designed for lowest emittance (at limited circumference), strong sextupoles for correction of large chromaticities generated by the required focusing, introduce significant nonlinearities into the lattice that have to be considered in MA calculations:

\footnotetext{
${ }^{1}$ The well-known integral formula assuming flat beams and nonrelativstic transverse motion agrees on a $10 \%$ level with detailed Monte Carlo simulations [8].
}

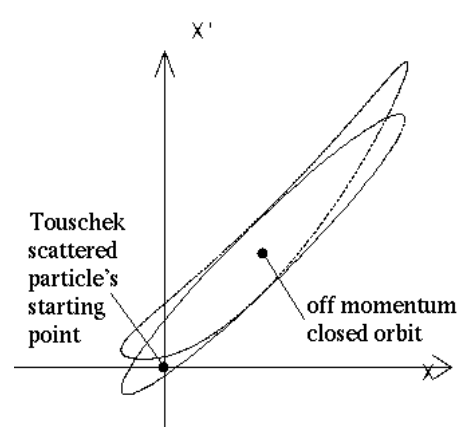

Figure 1: Nonlinear betatron motion: A Touschek scattered particle starting to oscillate around the off-momentum orbit would be accepted by the linear separatrix (ellipse) but not by the nonlinear separatrix.

- Momentum dependency of linear optics parameters: Calculations on light source lattices optimized for large MA [1] have to consider momentum deviations up to $\pm 10 \%$. Within this wide range the $2 \nu_{x}, 2 \nu_{y}$ chromatic resonance drive terms from the sextupoles' Hamiltonian cause momentum dependent beta beats and second order chromaticities [2].

- Nonlinear variation of closed orbit with momentum, i.e. higher order dispersion as included earlier in calculations at SOLEIL [11].

- Nonlinear betatron motion: Momentum dependent dynamic apertures smaller than the physical apertures or distortions of the transverse eigenfigures and mismatch to the physical apertures as illustrated in Fig. 1 lead to a reduction of local MA.

- Synchrotron oscillation: Due to higher order chromaticity scattered particles walk over wide regions in the tune diagram crossing several betatron resonances. Hence we observe a significant reduction of MA when including synchrotron oscillations compared to fixed$\delta$ calculations.

- Magnet alignment errors: Touschek lifetime depends on the emittance coupling factor $\kappa=\varepsilon_{y} / \varepsilon_{x}$. Including alignment errors generating nonzero $\kappa$ in a flat lattice is required to predict numbers for average lifetime and its variation for different error distributions ("seeds").

- Mini gap insertions: The beam halo has larger coupling than the beam core, as observed for example at ESRF [10], since large amplitude particles from scattering suffer more from higher order coupling resonances. For the performance of light sources using undulators with full gap heights as small as $4 \mathrm{~mm}$ it is essential to know how this affects the lifetime. 
In order to include all these effects from the Touschek lifetime point of view we take a brute force approach by starting particles from the beam core with some momentum deviation, i.e with the $6 \mathrm{D}$ initial vector $\left(x, p_{x}, y, p_{y}, \delta, \Delta s\right)=$ $(0,0,0,0, \pm \delta, 0)$ as it will be immediately after a Touschek scattering event [12]. Tracking and binary search for the maximum accepted $\delta$ gives the local MA. The resulting stepwise function of lattice MA $\delta_{a c c}^{L}(s)$ then is entered into the Touschek integral. If misalignments are to be included the calculation is repeated for a number of random seeds. This procedure was implemented into the program TRACY [5].

\section{MODEL PARAMETERS}

The SLS lattice in "D2A optics" as current reference lattice has dispersion free straight sections with mini- $\beta$-optics, and a natural emittance of $4.8 \mathrm{~nm}$ at $2.4 \mathrm{GeV}$. The working point was set to $20.82 / 8.28$ as a compromise between sextupole Hamiltonian suppression requirements [1] and vertical closed orbit distortion minimization. This is not the lattice mode with optimum dynamic aperture but still provides a TRELMA ${ }^{2}$ of $5.1 \%$ (fixed- $\delta$-calculation), which is larger than $3.9 \%$ provided by the $2.6 \mathrm{MV}$ RF system [7].

At SLS magnets are rigidly mounted onto girders, the girders are connected into a "train link" by means of horizontal sensor and hydrostatic levelling systems [3]. The following table gives alignment (horizontal \& vertical) and tilt errors considered as realistic (rms values, cut at $2 \sigma$ ):

\begin{tabular}{|l|c|c|}
\hline & shift $[\mu \mathrm{m}]$ & tilt $[\mu \mathrm{rad}]$ \\
\hline elements to girder & 50 & 100 \\
girder to girder & 100 & 0 \\
girder absolute & 300 & 25 \\
\hline
\end{tabular}

After setting the misalignments a SVD closed orbit correction was applied, preceded by a beam threader, if no initial orbit was found, and followed by an emittance coupling suppression by means of small skew quadrupoles [4]. The average coupling was $0.27 \%$ before and $0.10 \%$ after coupling suppression.

The narrow gap from insertion devices was included in tracking by reducing the vertical aperture on a length of $2 \mathrm{~m}$ in all six short straight sections $\left(\beta_{y}^{*}=1.6 \mathrm{~m}\right)$.

Tracking was done for 1.5 synchrotron oscillation periods (100...300 turns). Binary search resolution for determination of local MA was $0.01 \%$. All calculations assumed $1 \mathrm{nCb}$ single bunch charge.

\section{RESULTS}

\subsection{Touschek lifetime and $R F$ voltage}

Fig. 2 displays the Touschek lifetime, normalized to $\kappa=0.1 \%$, for the error free lattice in linear approximation

\footnotetext{
${ }^{2}$ The Touschek relevant effective lattice momentum acceptance is the MA value, where the RF alone (i.e. infinite lattice MA), set to the corresponding voltage, and the lattice alone (i.e. infinite RF MA) give equal Touschek lifetimes (normalized to bunchlength) [12]
}

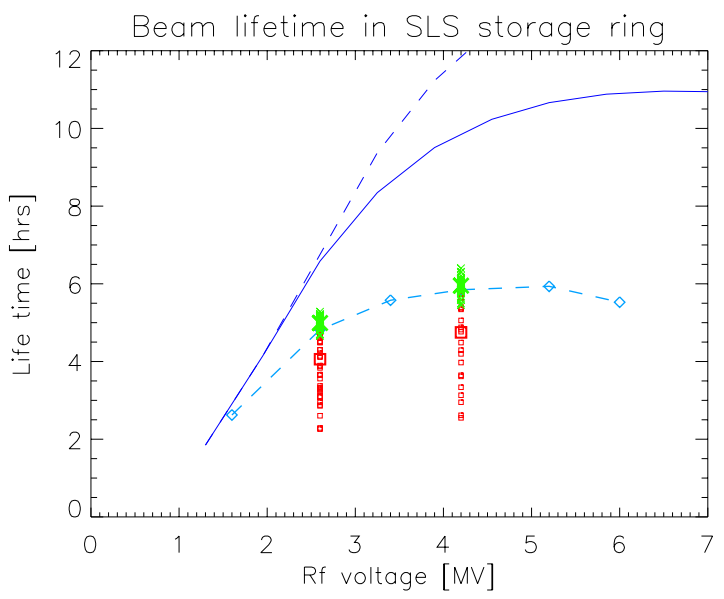

Figure 2: Touschek lifetime as a function of RF voltage for progressive model refinements:

linear lattice, no errors, fixed- $\delta$ (- - - )

adding lattice nonlinearities (- - )

adding synchrotron oscillation (- - --$)$

adding alignment errors, $\langle\kappa\rangle=0.27 \%$, normalized to $\kappa=0.1 \%(\square$ 口० $)$

adding coupling control, $\langle\kappa\rangle=0.1 \%,(\times, \times \times \times)$

according to eq.(1) and for the nonlinear optics in fixed- $\delta$ calculation as well as with synchrotron oscillation. Mini gaps were not yet set.

Already for the ideal lattice we see a strong reduction of lifetime, i.e. lattice MA (dynamic aperture), when including nonlinearities and synchrotron oscillation. Thus increasing the RF voltage beyond the planned 2.6 MV would gain only little in lifetime.

After introducing misalignments and closed orbit correction we observe large spread of different seeds and further $(\kappa$-normalized!) lifetime decrease, however after applying coupling suppression the ideal lattice values are restored and the spread from different seeds is compressed as shown for two voltage values in Fig. 2.

\subsection{Touschek lifetime and gap height}

Fig. 3 displays the Touschek lifetime, again normalized to $\kappa=0.1 \%$, as a function of the mini gap full height. The ideal lattice assuming $100 \%$ halo coupling would have reduced lifetime for full gap heights $\leq 10 \mathrm{~mm}$. Assuming $0.1 \%$ for both core and halo coupling the mini gaps show no effect. After introducing misalignment errors (fixed- $\delta$ calculation) we see that the halo coupling is larger than the core coupling but not at $100 \%$.

Including synchrotron oscillation seriously decreases lifetime for any gap height. However after coupling correction, the $\kappa$-normalized lifetime recovers and becomes almost independent of mini gap height.

Fig. 4 illustrates how lifetime limitations from the mini gap insertion are removed by coupling correction. 


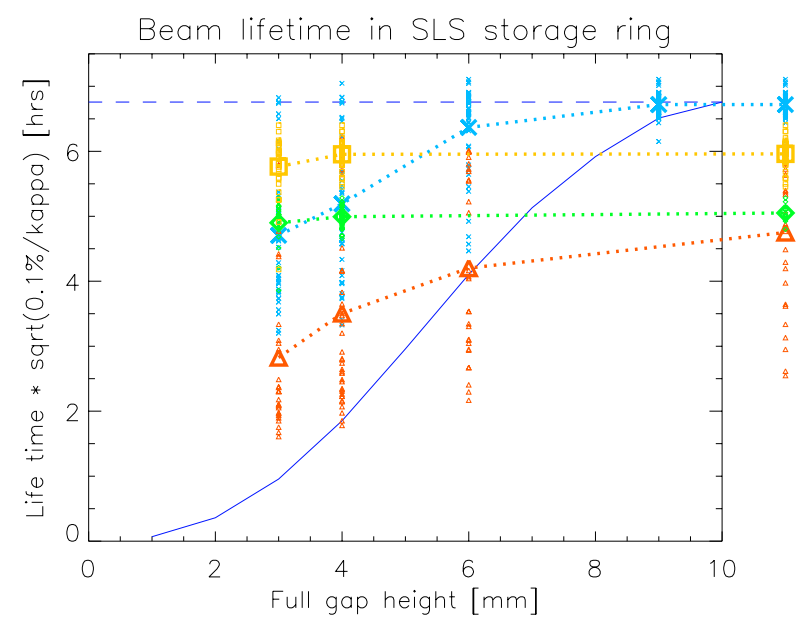

Figure 3: Touschek lifetime vs. insertion gap full height: Error free lattice for $\kappa_{\text {halo }}=100 \%(-)$ and $\kappa_{\text {halo }}=\kappa_{\text {core }}=0.1 \%(---)$, (nonlinearities included, fixed $\delta$ calculation, $V_{r f}=2.6 \mathrm{MV}$ )

Lattice with alignment errors, fixed- $\delta$ calculation, $V_{r f}=2.6 \mathrm{MV}(\cdots \times \cdots)$

Tracking with synchrotron oscillations, $V_{r f}=4.2 \mathrm{MV}$, $\langle\kappa\rangle=0.27 \%$, normalized to $\kappa=0.1 \%(\cdots \triangle \cdots)$

Application of coupling suppression, $\langle\kappa\rangle=0.1 \%$, for $V_{r f}=4.2 \mathrm{MV}(\ldots \square \ldots)$ and $2.6 \mathrm{MV}(\ldots \diamond \cdots)$

Points outside plot window are for a full "gap" height of $32 \mathrm{~mm}$, i.e. no insertions.
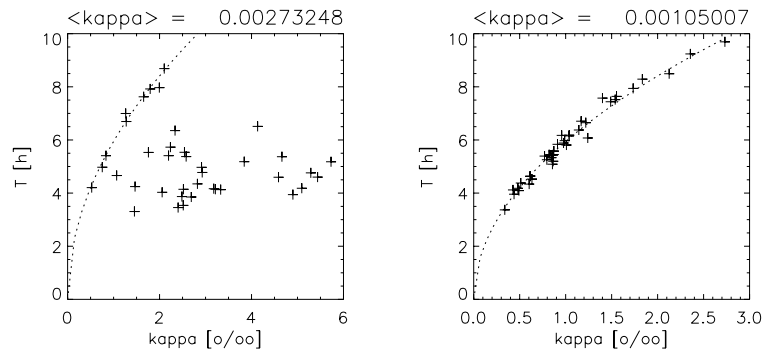

Figure 4: Touschek lifetime vs. emittance coupling factor $\kappa$ without (left) and with (right) coupling correction for different seeds of alignment error settings. Points not lying on the dotted $\mathrm{T} \propto \sqrt{\kappa}$ curve for the ideal lattice indicate beam losses at the insertion's $4 \mathrm{~mm}$ gap. Actually the average lifetime is higher with reduced coupling!

\subsection{Gas scattering lifetime}

Scattering of electrons on residual gas molecules also imposes serious lifetime restrictions: The cross section for electron losses after elastic scattering depends on the minimum transverse acceptance and on the vacuum conditions. With an insertion length of $\approx 2 \mathrm{~m}, \beta_{y}=2.2 \mathrm{~m}$ at entrance and exit as provided by the "D2A" optics of the SLS lattice is almost at optimum. Assuming 1 nTorr of carbon monoxide for the residual gas, the gas scattering lifetime obtained from the code ZAP [13] is $14 \mathrm{hrs}$ for $4 \mathrm{~mm}$ insertion full gap height.

\subsection{RF upgrade for lifetime improvement}

Increasing the RF voltage in order to raise the RF MA or installation of a $3^{\text {rd }}$ harmonic cavity for bunch lengthening are two options for increasing the Touschek lifetime. Higher voltage gains little lifetime, at least for the lattice mode investigated here. The harmonic cavity however, fighting Touschek effect at its origin instead of curing the consequences, would lengthen the bunch and correspondingly the Touschek lifetime by a factor of 4 and thus is the method of choice [9]. The following table displays results of Touschek and total lifetime assuming $4 \mathrm{~mm}$ gap insertions without and with a $3^{\text {rd }}$ harmonic cavity ${ }^{3}$ :

\begin{tabular}{|c|c|c|c|}
\hline $\begin{array}{c}V_{r f} \\
{[\mathrm{MV}]}\end{array}$ & $\begin{array}{c}\text { Touschek } \\
{[\mathrm{hrs}]}\end{array}$ & $\begin{array}{c}\text { Gas } \\
{[\mathrm{hrs}]}\end{array}$ & $\begin{array}{c}\text { Total } \\
{[\mathrm{hrs}]}\end{array}$ \\
\hline $2.6\left[+3^{r d}\right.$ harm.cav. $]$ & $4.8[19]$ & 14 & $3.6[8.1]$ \\
$4.2\left[+3^{r d}\right.$ harm.cav. $]$ & $5.9[24]$ & 14 & $4.2[8.8]$ \\
\hline
\end{tabular}

\section{CONCLUSION}

Mini gap insertion devices in the lattice partially reverse the usual dependency of Touschek lifetime on emittance coupling: suppression of the coupling factor to values as low as $10^{-3}$ gives optimum beam lifetime and removes sensitivity of the lifetime to particular alignment error settings while on the other hand providing high brightness. Larger emittance coupling does not increase the beam life time.

Increasing the RF voltage showed insignificant gain in lifetime, at least for the lattice mode studied here.

For SLS at standard operation conditions we expect a total lifetime of $3 \frac{1}{2} \mathrm{hrs}$ which may be increased to $8 \mathrm{hrs}$ by means of a $3^{\text {rd }}$ harmonic cavity.

\section{REFERENCES}

[1] J. Bengtsson et al., NIM A 404 (1998) 237

[2] J. Bengtsson, SLS-TME-TA-1997-0009, March 97

[3] M. Böge et al., Proc. EPAC-98, p.644

[4] M. Böge, M.Muñoz, A. Streun, these proceedings

[5] M. Böge, SLS-TME-TA-1999-0002, April 99

[6] H. Bruck, Accélérateurs circulaires de particules, Paris 1966

[7] M. E. Busse-Grawitz et al., these proceedings

[8] S. Khan, BESSY-TB 177/93

[9] P. Marchand, these proceedings

[10] G. Mülhaupt, priv. comm.

[11] A. Nadji et al., Proc. PAC 97, p.1517

[12] A. Streun, SLS-TME-TA-1997-0017, Nov. 97

[13] M. Zisman et al., ZAP User's Manual, LBL-21270, Dec. 86

${ }^{3}$ All lifetime numbers are defined for decay of beam current to half its initial value 